\title{
A NEW GENERAL SERIES OF BALANCED INCOMPLETE BLOCK DESIGNS
}

\section{RAMANUJACHARYULU}

0 . Summary. Let $v$ be any integer with $s_{1}$ the least prime power factor, the other prime power factors being $s_{2}, \cdots, s_{m}$. Assume $v$ is odd and consider the cartesian product of the $m$ Galois fields $\mathrm{GF}\left(s_{1}\right), \cdots, \mathrm{GF}\left(s_{m}\right)$ of orders $s_{1}, \cdots, s_{m}$ respectively. Let $x_{i}$ denote the primitive root of $\mathrm{GF}\left(s_{i}\right), i=1,2, \cdots, m$. Then labelling the points

$$
\alpha_{j+1}=\left(x_{1}^{j}, x_{2}^{j}, \cdots, x_{m}^{j}\right), \quad j=0,1, \cdots, s_{1}-2 ;
$$

and arbitrarily labelling the remaining points $\alpha$ of the product space, defining addition and multiplication of $\alpha$ 's coordinate-wise in their respective fields, we take the initial blocks.

$$
\begin{aligned}
& \left(0, \beta_{1} \alpha_{1}, \beta_{1} \alpha_{2}, \cdots, \beta_{1} \alpha_{k-1}\right) \\
& \left(0, \beta_{2} \alpha_{1}, \beta_{2} \alpha_{2}, \cdots, \beta_{2} \alpha_{k-1}\right) \\
& \vdots \\
& \left(0, \beta_{(v-1) / 2} \alpha_{1}, \beta_{(v-1) / 2} \alpha_{2}, \cdots, \beta_{(v-1) / 2} \alpha_{k-1}\right)
\end{aligned}
$$

where $k \leqq s_{1}$ if $m>1$ and $k<s_{1}$ if $m=1 ; 0=(0,0, \cdots, 0)$ and $\beta_{j}$ 's are such that no two $\beta_{j}$ 's add up to 0 (= the null vector): The theorem proved here is that by adding each of the points $\beta_{j}, j=0,1, \cdots, v-1$ of the product space to each of the above initial blocks we get a Balanced Incomplete Block Design with the parameters

$$
\left(v, \frac{v \cdot v-1}{2} \cdot \frac{k \cdot v-1}{2} \cdot k, \frac{k \cdot k-1}{2}\right)
$$

which is a new series generalising the series given by B. J. Gassner (Equal difference BIB designs, Proc. Amer. Math. Soc. 16 (1965), 378-380).

1. Introduction. Let $G$ be an abelian group of order $v$. A set of $k$ distinct elements of $G$ is called a difference set if the $k \cdot k-1$ differences of the elements of $D$ contain every nonzero element of $G, \lambda$ times. These definitions are generalised and in place of a single set $D$, one can take $t$ initial blocks of $k$ elements each where the $t \cdot k \cdot k-1$ differences from the $t$ blocks contain every nonzero element of $G$ the same number of times.

Received by the editors December 24, 1965. 
Let $\mathrm{GF}\left(s_{i}\right)$ denote a Galois field of order $s_{i}, i=1,2, \cdots, m$ and $x_{i}$ be a primitive root in the field. Let $v$ be an odd integer with the following prime power decomposition:

$$
v=p_{1}^{\theta_{1}} \cdots p_{m}^{e_{m}}=s_{1} \cdot s_{2} \cdots s_{m}
$$

where $s_{i}=p_{i}^{e} ; i=1,2, \cdots, m$.

Assume that $s_{1}$ is the least prime power factor of $v$ and let $\beta$ denote a general element of the cartesian product $G$ of the $m$ fields

$$
G=\mathrm{GF}\left(s_{1}\right) * \cdots * \mathrm{GF}\left(s_{m}\right) .
$$

Let us label some of the $\beta$ 's by $\alpha$ 's as follows:

$$
\begin{aligned}
\alpha_{j+1} & =\left(x_{1}^{j}, x_{2}^{j}, \cdots, x_{m}^{j}\right), \quad j=0,1, \cdots, s_{1}-2, \\
\alpha_{0} & =(0,0, \cdots, 0) .
\end{aligned}
$$

Let $B$ denote the set of points:

$$
B:\left(\alpha_{0}, \alpha_{1}, \cdots, \alpha_{k-1}\right)
$$

where $k \leqq s_{1}$ if $m>1$ and $k<s_{1}$ if $m=1$.

2. Some lemmas on $B$.

2.1. Lemma. Let $\alpha_{c}$ and $\alpha_{d}$ be any two distinct elements of $B$. Then $\alpha_{c}$ and $\alpha_{c}-\alpha_{d}$ have multiplicative inverses defined.

Proof follows easily since no coordinate of either $\alpha_{c}$ or $\alpha_{c}-\alpha_{d}$ is zero and hence a multiplicative inverse exists for each coordinate in their respective fields.

2.2. LemmA. If $\alpha_{c} \in B, c \neq 0,1$ and $m>1$, then $\alpha_{c}^{-1} \notin B$.

For, otherwise if a $d$ exists such that

$$
\alpha_{c} \alpha_{d}=\alpha_{c+d}=\alpha_{0}
$$

then

(2.2) $c+d=0 \bmod \left\{\left(s_{1}-1\right),\left(s_{2}-1\right),\left(s_{3}-1\right) \cdots,\left(s_{m}-1\right)\right\} \cdots$

since $c, d \leqq k_{1}-1 \leqq s_{1}-1$ and $c \neq d$, and the fields are all odd; $c+d$ can take at most the value $2\left(s_{1}-1\right)-1$. Thus if $c+d=s_{1}-1$ then $c+d$ $\neq 0 \bmod \left(s_{i}-1\right) i=2, \cdots, m$. Hence in no case can $(2.2)$ be satisfied.

2.3. Proposition. $A$ set $T$ of $(v-1) / 2$ points $\beta_{j}, j=1,2, \cdots$, $(v-1) / 2$ can be selected from the product space $G$ such that if $\beta_{j} \in T$, $-\beta_{j} \notin T$. 
3. Theorem. From the initial blocks

$$
\begin{aligned}
& B_{1}:\left(0, \beta_{1} \alpha_{1}, \beta_{1} \alpha_{2}, \cdots, \beta_{1} \alpha_{k-1}\right) \\
& B_{2}:\left(0, \beta_{2} \alpha_{1}, \beta_{2} \alpha_{2}, \cdots, \beta_{2} \alpha_{k-1}\right) \\
& \vdots \\
& \dot{B}_{(v-1) / 2}:\left(0, \beta_{(v-1) / 2} \alpha_{1}, \beta_{(v-1) / 2} \alpha_{2}, \cdots, \beta_{(v-1) / 2} \alpha_{k-1}\right)
\end{aligned}
$$

on adding $\beta_{j}, i=0,1,2, \cdots, v-1$ to each element of each block a balanced incomplete block design with the following parameters results in:

$$
\begin{aligned}
& v=v, \\
& b=v \cdot \frac{v-1}{2} \\
& r=k \cdot \frac{v-1}{2}, \\
& k=k, \\
& \lambda=k \cdot \frac{k-1}{2} .
\end{aligned}
$$

Proof. $\left\{\beta_{j}\right\}, j=0,1,2, \cdots, v-1$ are the $v$ elements of $G$ : the product space of the $m$ fields taken as treatments. First we establish that each initial block contains distinct elements then every two initial blocks are distinct if $m>1$ and finally that every treatment appears $r$ times and every pair of treatments appears $\lambda$ times.

If $B_{\boldsymbol{j}}$ had contained two identical points then we should have:

$$
\beta_{j} \alpha_{c}=\beta_{j} \alpha_{d}, \quad c \neq d \in\{1, \cdots, k-1\},
$$

i.e.

$$
\beta_{j}\left(\alpha_{c}-\alpha_{d}\right)=0 .
$$

Multiplying by $\left(\alpha_{c}-\alpha_{d}\right)^{-1}$ we should have $\beta_{j}=0$ which is not true. Hence $B_{j}$ contains distinct elements.

Consider

$$
B_{j}=\left(0, \beta_{j} \alpha_{1}, \cdots, \beta_{j} \alpha_{k-1}\right)
$$

and

$$
B_{i}=\left(0, \beta_{i} \alpha_{1}, \cdots, \beta_{i} \alpha_{k-1}\right)
$$

If $\beta_{j} \alpha_{1} \notin B_{i}$ then $B_{j}$ and $B_{i}$ are distinct blocks. If $\beta_{j} \alpha_{1} \in B_{i}$ then we 
show that $\beta_{i} \alpha_{1} \notin B_{j}$. Deny this and let $\beta_{j} \alpha_{1}=\beta_{i} \alpha_{c}, 1 \leqq c \leqq k-1$. Then $\beta_{i} \alpha_{1}=\beta_{j} \alpha_{c}^{-1} \alpha_{1}^{2}=\beta_{j} \alpha_{c}^{-1}\left(\alpha_{1}^{2}=\alpha_{1}\right.$ for $\alpha_{1}=\alpha_{1}^{2}=(1,1, \cdots, 1)$. But $\alpha_{c}^{-1} \notin B$ and hence $\beta_{i} \alpha_{1} \notin B_{j}$.

Thus the $b$ blocks are distinct, we will show that each treatment appears $r$ times. Let $\beta$ be any point in $G$. Consider the $v$ blocks generated by $B_{j}$ for some fixed $j, j=1,2, \cdots,(v-1) / 2$. Let

$$
\beta-\beta_{j} \alpha_{c}=\beta_{c} \text { for } c=0,1, \cdots, k-1
$$

then $\beta$ appears in the $k$ blocks $\left\{B_{j}+\beta_{c}\right\}, c=0,1, \cdots, k-1$. Hence as $j=1,2, \cdots,(v-1) / 2$ we observe that every treatment appears in $r=k-(v-1) / 2$ blocks.

Now we proceed to determine $\lambda$. Consider any two points $\beta_{1} \alpha_{c}$ $\neq \alpha_{d} \beta_{1} \in B_{1}$. Let $\beta_{1}\left(\alpha_{c}-\alpha_{d}\right)=\beta_{1} \alpha$. Then in the initial blocks $B_{j}$ the corresponding difference is $\beta_{j} \alpha$. The differences $\left\{\beta_{j} \alpha,-\beta_{j} \alpha\right\} j=1,2$, $\cdots,(v-1) / 2$ are all distinct. For if $\beta_{j} \alpha=\beta_{j^{\prime}} \alpha$ then multiplying by $\alpha^{-1}$ we should have $j=j^{\prime}$ or if $\beta_{j} \alpha=-\beta_{j^{\prime}} \alpha$ then again $\left(\beta_{j}+\beta_{j^{\prime}}\right)=0$ which is not true by choice. Thus in the initial block the differences between $c$ and $d$ elements produce all the nonzero elements of $G$ exactly once. Given two points $\beta_{i}$ and $\beta_{j}$ let $\beta_{i}-\beta_{j}=\beta$ say. In the initial block choose any two distinct points $\alpha_{c}$ and $\alpha_{d}$ then there exists a unique $\beta_{l}, l=1,2, \cdots,(v-1) / 2$ such that

$$
\beta_{l} \alpha_{c}-\beta_{l} \alpha_{d}=\beta,
$$

since $\pm\left(\alpha_{c}-\alpha_{d}\right) \beta_{t}, t=1,2, \cdots,(v-1) / 2$ gives all nonzero $\beta$ 's exactly once. In the set of $v$ blocks generated by $B_{l}$ then, $\beta_{i}$ and $\beta_{j}$ occur together in exactly one block. Since we have $C_{k, 2}$ pairs of $\left(\alpha_{c}, \alpha_{d}\right)$ every pair of treatments appears in $k \cdot k-1 / 2$ blocks.

An example of $v=9, b=36, r=16, k=4, \lambda=6$, constructed using the 4 initial blocks

$$
\begin{gathered}
(0,1,-1, x) ;(0, x,-x,-1) ;(0, x+1,-x-1, x-1) ; \\
(0, x-1,-x+1,-x-1) ;
\end{gathered}
$$

in the field $\mathrm{GF}\left(3^{2}\right)$ with the irreducible function $x^{2}+1=0$ :
$\left(\begin{array}{llllllll}1 & 2 & 3 & 4\end{array}\right)\left(\begin{array}{llll}2 & 3 & 1 & 6\end{array}\right)$
(3 1125$)$
(1 45
3) $\left(\begin{array}{llll}2 & 6 & 9 & 1\end{array}\right)$
$\left(\begin{array}{llll}3 & 8 & 7 & 2\end{array}\right)$
$\left(\begin{array}{lllllll}1 & 6 & 7 & 8\end{array}\right)\left(\begin{array}{llll}2 & 8 & 4 & 5\end{array}\right)$
(3 $\left.\begin{array}{llll}3 & 4 & 9 & 6\end{array}\right)$
$\left(\begin{array}{llll}1 & 8 & 9 & 7\end{array}\right)$
$\left(\begin{array}{llll}2 & 4 & 5 & 7\end{array}\right)$
(3 $\left.6 \begin{array}{lll}3 & 5 & 9\end{array}\right)$
$\left.\begin{array}{llll}(4 & 6 & 8 & 5\end{array}\right)\left(\begin{array}{llll}5 & 9 & 7 & 1\end{array}\right)$

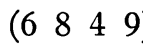
$\left(\begin{array}{llll}4 & 5 & 1 & 8\end{array}\right)$
$\left(\begin{array}{llll}5 & 1 & 4 & 7\end{array}\right)$

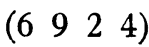
$\left(\begin{array}{llll}4 & 9 & 3 & 7\end{array}\right)\left(\begin{array}{llll}5 & 2 & 8 & 3\end{array}\right)$
$\left(\begin{array}{llll}6 & 7 & 1 & 5\end{array}\right)$
(4 $\left.\begin{array}{llll}4 & 7 & 2 & 3\end{array}\right)$
(5 $\left.3 \begin{array}{lll}5 & 6 & 8\end{array}\right)$
$\left(\begin{array}{llll}6 & 5 & 1 & 3\end{array}\right)$
$\left.\begin{array}{llllll}(7 & 5 & 9 & 3\end{array}\right)\left(\begin{array}{llll}8 & 4 & 6 & 7\end{array}\right)$
$\left.\begin{array}{llllll}(9 & 7 & 5 & 2\end{array}\right)\left(\begin{array}{llll}7 & 3 & 8 & 9\end{array}\right)$
$\left(\begin{array}{llll}8 & 7 & 3 & 6\end{array}\right)$
$\left(\begin{array}{llll}9 & 2 & 6 & 5\end{array}\right)$
$\left(\begin{array}{llll}7 & 1 & 6 & 2\end{array}\right)$
) $\left(\begin{array}{llll}8 & 5 & 2 & 9\end{array}\right)$
(9 $\left.\begin{array}{llll}9 & 4 & 1\end{array}\right)$
$\left(\begin{array}{llll}7 & 2 & 4 & 6\end{array}\right)$
(8) 91
2) $\left(\begin{array}{llll}9 & 1 & 8 & 4\end{array}\right)$ 


\section{REFERENCES}

1. R. C. Bose, On the construction of balanced "Incomplete block designs," Ann. Eugenics, 9 (1939), 358-399.

2. L. E. Dickson, Linear groups with an exposition of Galois Field Theory, Dover, New York, 1958.

3. B. J. Gassner, Equal difference BIB designs, Proc. Amer. Math. Soc. 16 (1965), 378-380.

Indian Statistical Institute, Calcutta 